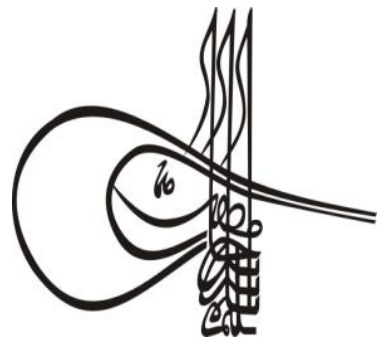

Received/Geliș: 01.04.2019

\section{Turkísh Studies Educational Sciences}

Volume 14 Issue 3, 2019, p. 963-973

DOI: 10.29228/TurkishStudies.22913

ISSN: 2667-5609

Skopje/MACEDONIA-Ankara/TURKEY

Research Article / Araștırma Makalesi

ArticleInfo/Makale Bilgisi

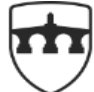

INTERNATIONAL BALKAN UNIVERSITY

EXCELLENCE FOR THE FUTUR IBU.EDU.MK

This article was checked by intihal.net.

\title{
DIE ANWENDUNG AUTHENTISCHER TEXTE IM FREMDSPRACHLICHEN DEUTSCHUNTERRICHT
}

\author{
Hasan YILMAZ*
}

\begin{abstract}
ZUSAMMENFASSUNG
Der Hauptzweck der Fremdsprachenkurse besteht darin, die kommunikative Kompetenz der Studierenden zu entwickeln. Der gleichberechtigten Entwicklung der vier grundlegenden Sprachkenntnisse sollte große Aufmerksamkeit gewidmet werden. Wie bekannt, haben die Sprachkenntnisse in der täglichen Kommunikation keinen Vorrang vor anderen. $\mathrm{Zu}$ diesem Zweck sollten in allen Fremdsprachenkursen vorrangig schriftliche oder mündliche authentische Texte verwendet werden. Welche Texte können auch als authentische Texte betrachtet werden? Texte, die die Zielkultur als vielseitig widerspiegeln, werden als authentische Texte bezeichnet. Die authentischen Texte werden also nicht für den Fremdsprachenunterricht konzipiert. In diesem Sinne haben Fremdsprachenlehrer eine große Verantwortung. Es ist die erste Aufgabe des Lehrers, authentische Texte in die Klasse mitzubringen, die dem Fremdsprachennieau des Schülers entsprechen. Die authentischen Texte eignen sich gut für die Entwicklung der jeweiligen Sprachkenntnisse. Diese Texte, die die alltägliche Sprache beinhalten, sind äußerst nützlich für die Entwicklung der jeweiligen Sprachkenntnisse. Sie können daher problemlos in allen Fremdsprachenkursen eingesetzt werden. In dieser Studie werden Online-Zeitungstexte als authentische Texte unter die Lupe genommen, die im Fremdsprachenunterricht eine wichtige Rolle spielen und durch technologische Entwicklungen leicht zugänglich sind. Zeitungstexte enthalten universelles Vokabular, da sie sich auf allgemeine Themen beziehen. Die Studierenden, die sich mit diesen Texten aus ihrem täglichen Leben auskennen, können ihre früheren Kenntnisse nutzen, um den Text zu verstehen. In dieser Studie wurden einige Erklärungen gegeben, wie diese Texte in Fremdsprachenkursen verwendet werden
\end{abstract}


können. Diese Texte können in neun verschiedenen Abschnitten des Unterrichts behandelt werden, und der Lehrer kann diese Abschnitte selbst gestalten. Diese Texte werden auch sehr nützlich für die Bereicherung des Vokabulars angesehen.

Schlüsselwörter: Fremdsprachenunterricht, Sprachkenntnisse, authentische Texte, Zeitungstexte, Kommunikation.

\title{
USE OF ORIGINAL TEXTS IN GERMAN AS A FOREIGN LANGUAGE
}

\begin{abstract}
The main purpose of foreign language courses is to develop the communicative competence of the students. Great attention should be paid to the equal development of the four basic language skills. As it is known, in daily life communication, no language skills are superior to each other. For this, written or verbal authentic texts should be used as priority in all foreign language courses. Which texts can be also considered as authentic texts? Texts that reflect the target culture as multi-faceted are called authentic texts; in other words, the authentic texts are not created for foreign language teaching. In this sense, foreign language teachers have a great responsibility. It is the first responsibility of the teacher to find authentic texts appropriate to the foreign language level of the student. The authentic texts are well suited for the development of each language skill. These texts, which include the daily spoken language, are extremely useful for the development of each language skills. Therefore, they can be used easily in all foreign language courses. In this study, online newspaper texts as authentic texts which have an important place in foreign language teaching and which can be easily accessed thanks to technological developments are discussed. Newspaper texts contain universal vocabulary as they refer to universal issues. The students who are familiar with these subjects from their daily life can use their prior knowledges to understand the text. In this study, some explanations have been made on how these texts can be used in foreign language courses. These texts can be examined in nine different sections in the lessons, and the teacher can shape these sections themselves. These texts are also known to be very useful in terms of the development of the vocabulary.
\end{abstract}

\section{STRUCTURED ABSTRACT}

The general purpose of foreign language teaching is to develop the basic language skills and to enable the students to come to the qualification to communicate verbally and written in the target culture environment. In foreign language teaching, all foreign language students also want to achieve communicative competence for this purpose. What, then, means communicative competence in foreign language teaching? Communicative competence means that students express themselves in a meaningful and correct way in the target culture, both written and verbally. In order to achieve this aim, four basic language skills need to

Turkish Studies - Educational Sciences

Volume 14 Issue 3, 2019 
be developed together in foreign language teaching process. In the communicative situations in our native language, as we already know from daily life, there is no priority or superiority of any language skills during communication.

In order to be able to learn a foreign language, students should be familiar with the current language in the target culture. Today's technology such as TV, Internet, Mp3 offers such facilities.

For this reason, it is very important that the students encounter the authentic language used in the target culture and not an artificial language in foreign language courses. Authentic texts are actually produced for daily communication. Such texts are not texts written to teach a foreign language.

Online newspapers, which offer sections from the daily life of foreign languages, are among the authentic texts that reveal the target culture from different directions. Such texts that contain current words and structures can be included in every lesson. In this sense, newspaper news texts considered comparatively in the lessons are among the most important authentic sources that naturally improve students' vocabulary.

The aim of this study is to express the contribution of news in newspapers to the development of basic language skills in foreign language teaching. This study also aims to show in a concrete way that newspaper texts make a significant contribution to linguistic competences of students. In the case of a newspaper text of target culture, it was shown how such texts can be used in foreign language courses.

In this study, we focus on one of the informative texts and the use of news for teaching foreign languages in lessons. Such newspaper texts are considered to be more appropriate for the development of language skills of foreign language students. Because the information contained in the news is related to daily life and creates more interest among students. Students can also compare the content of this news with their native language newspaper texts. In this way, students will be familiar with the content of the text in foreign language because they have met the texts written in their native language in their daily lives. In addition, this kind of text gives students the opportunity to get to know the target culture better. Such texts help students learn something new in terms of cultural studies.

Students will also have the opportunity to compare their culture and target culture through the newspaper's text on the same topic, especially with newspapers in their native language. Thus, they will see similarities and differences between the two cultures, and their intercultural competences will also develop. Taking the same subjects from different newspapers with the target language will mean that the students will be enriched in the vocabulary, because they will see that the same text content can be expressed in different words and expressions in a foreign language.

The fact that students encounter other unknown words while trying to find the meaning of unknown words in a text should be considered as an important contribution to the process of foreign language teaching.

Turkish Studies - Educational Sciences

Volume 14 Issue 3, 2019 
The use of monolingual dictionaries to find the meaning of unknown words should be particularly recommended by the teacher. Because students will learn new words while searching for the meaning of an unknown word. Exercises will also increase the benefits of such texts in foreign language teaching. In particular, the following points should be noted:

- try to understand text titles,

- comparison of native language texts with texts written in foreign language,

- to concentrate on the news or message what it actually wants to give,

- try to understand unknown words with the help of the context before.

In short, newspaper texts do not only improve reading skills of learners in foreign language courses. The use of texts in newspapers and news articles in other language courses will also help to develop other basic language skills. Main purpose is to have a communicative ability in a foreign language. In order to achieve this aim, it is a necessity to get the subject of newspaper texts and to recognize the daily language of the target culture with these texts.

Keywords: Foreign language teaching, language skills, authentic texts, newspaper texts, communication.

\section{Einleitung}

Das Hauptziel eines fremdsprachlichen Lehr- und Lernprozesses ist es, dass die Lerner die Fremdsprache beherrschen, die sie sich zu lernen bemühen; anders formuliert, sie möchten über sprachliche Fertigkeiten verfügen, die sie zur mündlichen und schriftlichen Kommunikation in den zielkulturellen Sprachsituationen führen. Alle Fremdsprachenlerner möchten in einer Fremdsprache die so genannte kommunikative Kompetenz erreichen. Was bedeutet also kommunikative Kompetenz in der Fremdsprachendidaktik? „Kommunikative Kompetenz” heisst, dass die Lerner dazu befähigt werden, sich in der Zielkultur situationsadäquat und sinngemäss -sowohl schriftlich als auch mündlichäussern zu können. Um diesem Ziel gerecht zu werden, ist es aber notwendig, dass die vier Grundsprachfertigkeiten zusammen gefördert werden. Dabei geht man von der Annahme aus, dass keine Sprachfertigkeit Vorrang hat, wie es uns schon im muttersprachlichen Alltag bekannt ist.

Es gibt heutzutage viele technische Mittel (Tv, Internet, Mp3 usw.), die es den Lernenden ermöglichen, sich mit der aktuellen Sprache zu befassen. Die erste Voraussetzung, eine Fremdsprache zu erlernen, bzw. zu beherrschen, ist, dass man -auf dem Wege des Lernprozesses- sich nicht mit der künstlichen Sprache sondern mit der alltäglichen, also mit der in der Zielkultur verwendeten, authentischen Sprache beschäftigt. Authentische Texte, die eigentlich für alltägliche Kommunikation konzipiert sind, gehören zu den Textarten, die neben der Förderung anderer Grundsprachferigkeiten zur Wortschatzbereicherung bei den Lernenden dienen (Ghaffar1, 2017, s. 11). Dies ist aber erst dann möglich, wenn den Lernenden aktuelle und authentische Mittel, die das Realleben der Zielsprache vielseitig vor Augen führen, zur Verfügung gestellt werden. Auch die Online- Zeitungen gehören zu solchen Mitteln, mit denen sich die Lerner auseinandersetzen sollen, damit sie die aktuelle Sprache näher kennenlernen und sich auf dem Wege zur Förderung sprachlicher Grundfertigkeiten entwickeln können. Es erübrigt sich zu unterstreichen, dass das Können einer Fremdsprache eigentlich von den 
Wörtern abhängen (Büyükahıska ve Çebi Kozallı, 2018, s. 310). Ohne genügende Wortschatzkenntnisse ist es fast unmöglich, in der Fremdsprache kommunizieren zu können. Bei der Bereicherung des Wortschatzes haben deshalb authentische Texte von grossem Belang, weil sie sehr aktuelle Wörter beinhalten. Auch die Zeitungstexte gehören unumstritten zu solchen authentischen Texten.

Ziel dieses Beitrags liegt deshalb darin, auf konkrete Weise vorzulegen, inwieweit die Nachrichten in den Zeitungen im Rahmen der Förderung der Teilfertigkeiten einen grossen Beitrag zur Sprachkompetenz der Lernenden leisten. Bevor ich ins Thema einsteige, wäre es angebracht, kurz zu erläutern, aus welchen Texten die Zeitungen bestehen. Dabei habe ich von Lügers Typologie Gebrauch gemacht, die bezüglich der Pressetexte vielen Wissenschaftlern als Wegweiser dienen.

Wie H.-H. Lüger. (1995, s. 65). zum Ausdruck bringt, haben Pressetexte sowohl viele Gemeinsamkeiten als auch Unterschiede, die bei der Entstehung vieler heterogenen Texte mitwirken und deshalb eine Typologie voraussetzen. Lüger unterscheidet fünf journalistische Textklassen nach dem Kriterium der Intentionalität. Er versucht also die Pressetexte, nach deren Funktion und Intention zu unterscheiden. Was bezweckt man eigentlich mit den betreffenden Texten? Welche kommunikative Absicht haben sie? Nach seiner Typologie sind die journalistischen Textsorten in fünf Grundklassen zu gliedern:

- Kontaktorientierte Texte: Boulevard-Texte

- Informationsbetonte Texte: Meldung, weiche Nachricht, harte Nachricht, Bericht Reportage, Wetterbericht und Sachinterview

- Meinungsbetonte Texte: Kommentar, Glosse, Kritik, Meinungsinterview

- Auffordernde Texte: Gesuch, Werbung

- Instruierend-anweisende Texte: Handlungsanleitungen, Ratgebungen.

Diese Studie konzentriert sich nur auf die Anwendung der Meldungen und Nachrichten, die nach Lügers Typologie zu den informationsbetonten Texten zählt. Der Grund für so eine Beschränkung besteht darin, dass man sich in den Unterrichtssituationen mit solchen Texten befasst hat, da sie für noch geeigneter zur Förderung der Sprachfertigkeiten unserer Zielgruppe angesehen wurde. Denn die Informationen in den Nachrichten sind alltagsbezogen und erwecken mehr Interesse bei den Lernenden, weil sie den Inhat dieser Nachrichten mit dem ihrer muttersprachlihen Zeitungen vergleichen können. Dadurch können sie beim Entschlüsseln der Nachrichten diesbezügliches Vorwissen aktivieren. Ausserdem bietet diese Art von Texten den Lernenden die Gelegenheit, die Zielkultur näher kennenzulernen. Diese Textsorten dienen dazu, dass sich die Lerner bezüglich der Landeskunde etwas neues lernen.

\section{1- Zeitungsnachrichten als informationsbetonte Texte}

In Pressetexten dieses Typs werden primär Informationen über Sachverhalte vermittelt. Es fehlen hier eigene Einstellungen und Bewertungen des Journalisten oder sie stehen nicht im Vordergrund. Solche Texte sind für den fremdsprachlichen Deutschunterricht noch geeigneter. Denn sie ermöglichen dem Lernenden, den Text leichter zu dekodieren, weil sie aktuell sind. Das zu behandelnde Thema ist dem Lernenden aus seinem Alltag höchstwahrscheinlich schon bekannt. Ein anderer wichtiger Grund für die vorrangige Behandlung solcher Textsorten ist es, dass sie durch die alltäglichen Wörter und Wendungen den Wortschatz des Lernenden bereichern. Die Sätze, die in den Texten vorkommen, finden auch im Alltag der Zielkultur öfters Anwendung. Die informationsbetonten Texte in den Zeitungen sind folgenderweise aufzuzählen: 


\section{- Meldung}

Diese Textsorte besteht aus einer einfachen Sachverhaltsdarstellung. Es ist bekannt, dass man sich in solchen Texten nur auf die Vermittlung von Fakten konzentriert. Die Zusammenhänge oder Hintergründe des behandelten Themas werden nicht erwähnt. Sie spielen bei der Darstellung fast keine Rolle. Die für Meldungen charakteristische sprachliche Handlung ist die Mitteilung. Deshalb kann eine Meldung auch nur aus einem einzigen Satz bestehen. Viele verschiedene Ereignisse aus dem Alltag werden in dieser Textsorte behandelt, so dass das Themenspektrum von politischen Sachverhalten bis zu sportlichen Aktivitäten auf der Welt reicht.

\section{- Harte Nachricht}

Diese Art von den journalistischen Nachrichten wird als die „Urzelle“ der Zeitung bezeichnet. Man erwartet von solchen journalistischen Textsorten, dass sie den Leser aktuell, sachlich (ohne Beigabe von Kommentierungen) und prägnant informieren. Es ist auch hinzuzufügen, dass der Textaufbau der harten Nachricht einige spezifische Merkmale aufweist. Man geht im allgemeinen Sinne von einer zentralen (Kern)aussage aus; dann die Zusatzinformationen und Erweiterungen können dieser Hauptinformation folgen. Die für den Leser als interessant angesehene Kenntnis steht im Titel. Durch den Fettdruck des Satzes im Vorspann (Lead) versucht man, das Leseinteresse zu erwecken. Nach dem Prinzip abnehmender Wichtigkeit sind die Zusatzinformationen im Haupttext (Body) zu lesen. Es ist aber auch die Aufmerksamkeit darauf zu lenken, dass es bestimmte Ähnlichkeiten mit der Textsorte "Meldung" gibt; die grösste Ähnlichkeit besteht darin, dass auch beide Nachrichtentypen nicht in chronologischer Reihenfolge über ein Ereignis berichten. In syntaktischer Hinsicht hat die harte Nachricht eine komplizierte Satzstruktur. Man bemüht sich also nicht darum, dass der Text für alle Leser verständlich ist; deshalb beansprucht man vom Leser das Allgemein- oder Vorwissen. Asserdem ist es auch in solchen Textsorten kennzeichnend, dass es um ziemlich längere Sätze geht.

\section{- Weiche Nachricht}

Als Gegenstand werden keine politischen oder wirtschaftlichen Ereignisse ausgewählt, sondern auf die Themen werden Gewicht gelegt, die mehr Neugierde und Interesse bei den Lesenden erwecken. $\mathrm{Zu}$ solchen Stoffen zählen vor allem Skandale, Naturkatastrophen, Verbrechen oder Ausschnitte aus dem Leben berühmter Persönlichkeiten.

Eine lesewerbende Darstellungsweise ist in dieser Textsorte auffällig. Um diese Darstellungsweise zu verwirklichen, werden aus folgenden sprachlichen Mitteln Nutzen gezogen:

- die temporale Strukturierung des vermittelten Geschehens,

- die Hervorhebung der Gegensätze bezüglich des Inhaltes,

- der Einsatz rhetorischer Figuren wie z. B. Parallelismus, Alliteration,

- verschiedene Modifikationen von Phraseologismen,

- interessante Wortspiele, suggeriert,

- die Bevorzugung direkter Rede, die die Authentizität bewirkt und Nähe zum Geschehen

- die Nutzung der nicht-ernsten, ironischen Ausdrücke und Signale.

\section{- Bericht}

Der Bericht wird meistens als eine Modifikation der harten Nachricht angesehen und bezeichnet. Im Gegensatz zu den harten Nachrichtentexten sind die Berichttexte im allgemeinen noch komplexer und vielfältiger. Ein bestimmter Sachverhalt steht im Mittelpunkt und darüber wird in chronologischer 
Reihenfolge informiert. Der Textaufbau kann weitere Komponenten aufweisen: Die zitierten Sätze, die Stellungnahme des Journalisten zu den Hintergrundinformationen eines Ereignisses, die den zum Thema gemachten Fall noch besser zu verstehen dienen usw.

\section{- Reportage}

Diese Darstellungsform informiert über eine räumlich und zeitlich begrenzte Geschichte. Obwohl sie viel Gemeinsames mit der Textsorte "Bericht" hat, zeigen sich bei näherer Betrachtung spezifische Unterschiede hinsichtlich der Informationspräsentation; in der Reportage werden das Geschehen und die Situation stark persönlich dargestellt. Der Reporter bewertet den Fall aus dem Augenblick des Erlebens. Damit wird bezweckt, einen möglichst breiten Leserkreis anzusprechen und aufzurütteln. Daher kommt es, dass diese Art der Zeitungstexte umgangsprachlich geprägt, syntaktisch einfach und leicht verständlich ist.

\section{2- Ein Unterrichtskonzept mit den authentischen Sprachprodukten am Beispiel der} Zeitungstexte

Im folgenden wurde durch einen schon im Unterricht behandelten Text einer Online- Zeitung deutlich gemacht, wie man mit den authentischen Texten und zwar mit den Nachrichten im fremdsprachlichen Deutschunterricht umgehen kann, damit die Lerner über die kommunikative Kompetenz verfügen können:

Vor dem Beginn des Kurses wird die Klasse in Gruppen eingeteilt. Jede Gruppe besteht aus höchstens 6 Personen. Ihre Aufgabe ist es, in 5 Minuten ihren Auftrag zu erledigen, so dass sie auch ihre Vermutungen in Bezug auf den Inhalt schriftlich festlegen können. Besonders in den Fächern, die zur Entfaltung des Leseverstehens und der Sprechfertigkeit dienen, sind die Anwendung solcher Texte sehr geeignet. Als Sozialform bei solchen Unterrichtseinheiten steht die Gruppenarbeit im Vordergrund. Ziel ist es, dass die Lerner mit Zusammenarbeit miteinander und voneinander lernen.

\section{Phase I:}

Es wird von dem Lehrenden besonders ein aktuelles Thema ausgewählt und in die Klasse mitgebracht, damit die Lerner ihr Vor- oder Allgemeinwissen aktivieren, bzw. von ihr Gebrauch machen können.

Aus unterrichtlichen Erfahrungen ist es mir als Lehrkraft schon bekannt, dass die Lerner mehr Interesse am aktuellen Weltgeschehen zeigen. Auch die schon befragten Lernerinteresse bestätigen unsere diesbezügliche Überzeugung.

\section{Textbeispiel aus dem zielkulturellen Alltag (ein Text aus einer Online - Zeitung)}

\section{„,Wassernot ist eine der größten Krisen unserer Zeit}

Wasser ist eine der wichtigsten Ressourcen weltweit. Doch die steigende Zahl Menschen verbraucht immer mehr. Experten sind sich sicher, dass sich Konflikte wegen Wassermangels künftig häufen werden.

In Deutschland vergisst man leicht, dass manche Dinge nicht selbstverständlich sind. Dass man beispielsweise nicht überall auf der Welt zu jeder Tages- und Nachtzeit einfach den Wasserhahn aufdrehen kann und dass das Nass dann zuverlässig und sauber fließt. Hierzulande verbraucht jeder Deutsche 120 Liter Wasser pro Tag. Man verbraucht nicht nur Wasser in Wasserform, sondern auch das indirekte Wasser.

Für ein Rindersteak sind beispielsweise 2000 Liter Wasser nötig, bevor es auf dem Teller landet, 20 Liter braucht man für 100 Gramm Gemüse. Die Landwirtschaft verbraucht 70 Prozent des weltweit genutzten Wassers; in den am wenigsten entwickelten Ländern liegt die Quote sogar bei 90 Prozent. 
Doch längst gibt es nicht genügend Wasser für alle. Derzeit haben laut Experten 750 Millionen Menschen keinen Zugang zu sauberem Trinkwasser. Und die Zahl dieser Menschen steigt. So werden nach aktuellen Prognosen Ende des Jahrhunderts zwei Milliarden Menschen unter direktem Wassermangel leiden. Konflikte sind programmiert.

Wo die Dürren zuschlagen

Besonders stark betroffen sind Länder in Afrika, Asien oder Lateinamerika. Sie kämpfen schon lange mit Dürren oder Überschwemmungen. Derzeit trifft es Äthiopien besonders hart; das Land am Horn von Afrika durchlebt wegen des Wetterphänomens „El Niño" schon das zweite Dürrejahr in Folge. Laut einem Bericht des Magazins „Science Advances “leiden weltweit vier Milliarden Menschen mindestens in einem Monat des Jahres unter Wasserknappheit, fast die Hälfte von ihnen in China und Indien. “(Online I)

\section{Phase II:}

Die Lehrkraft verlangt vor der Lektüre der Nachricht von den Lernern, dass sie mit Hilfe vom Titel vorhersehen, wovon im Text die Rede sein könnte. Manche Überschriften bestehen aus langen und deutlichen Sätzen, wie es sich am obigen Beispiel zeigen lässt. In solchen Fällen ist die Auswahl des Textes von der Lehrkraft abhängig. Sie kann das Schwirigkeitsgrad des Textes (auch den Kopf des Textes) selbst bestimmen. Kurz gesagt wird auf solche Weise die Klasse mental auf den Text vorbereitet und sie werden durch dieses Verfahren vor dem Anfang mit dem Entschlüsseln des Textes vorentlastet.

\section{Phase III:}

Jeder Gruppensprecher liest die Annahme seiner Gruppe über den eventuellen Inhalt des Textes vor. Die Lehrkraft macht sich inzwischen Notizen, welche Gruppe den Inhalt des Textes richtig vorgesehen hat.

Um diese Phase je nach dem fremdsprachlichen Niveau der Klasse noch komplizierter zu gestalten, kann sich die Lehrkraft für einen Text mit dem Titel entscheiden, der den Inhalt des Textes nicht so einfach verrät.

\section{Phase IV:}

Danach fordert die Lehrkaft die Studenten dazu auf, den Nachrichtentext in Gruppen zu Ende zu lesen. Bei dieser Beschäftigung dürfen die Lernenden das einsprachige Wörterbuch (Deutsch Deutsch) zu Rate ziehen, wenn sie auf unbekannte Wörter stossen. Das eigentliche Ziel dieser Verfahrensweise ist, dass die Lerner neue, deutsche Fremdwörter lernen, während sie die Bedeutung der unbekannten Wörter im Text herauszufinden versuchen. Das Nachschlagen im einsprachigen Wörterbuch dient ausserdem zur Einprägung der schon bekannten Wörter und Wendungen.

\section{Phase V:}

Nach dem Ende der Lektüre verlangt der Lehrende von den Gruppen, dass sie den Text ins Türkische übersetzen. Auch bei dieser Tätigkeit wird nur der Umgang mit einem deutschsprachigen Wörterbuch erlaubt. Wenn der Lehrende bei der Gruppenarbeit, während der schon bestimmten Zeit festlegt, dass sie z. B. die Bedeutung eines Fremdwortes im Türkischen nicht wiedergeben können, kann er sie dabei unterstützen. Durch so eine Verfahrensweise wird es den Lernenden zur Gewohnheit, die Bedeutungen unbekannter Wörter immer durch deutschsprachige Wörterbücher zu entschlüsseln. Auf solche Weise lernen sie neue Wörter kennen.

\section{Phase VI:}

Nach der Übertragung wird die türkische Wiedergabe der Nachricht von den verschiedenen Gruppenteilnehmern vor der Klasse vorgetragen. Die Lehrkraft trifft die Entscheidung, welche 
Mitglieder der Gruppen für den Vortrag der Übersetzung vor der Klasse verantwortlich sind. Ziel bei der Bestimmung eines anderen Gruppenteilnehmers als Sprecher ist, dafür zu sorgen, dass jeder aktiv an diesem Prozess teilnimmt und dass auf solche Weise die Hemmschwelle bei den manchen Lernenden überschritten wird. Auch hinsichtlich der Aufrechterhaltung der Konzentration der Gruppenarbeit und die Entwicklung der Gruppenzugehörigkeit ist es von eminenter Bedeutung, dass bei jeder unterrichtlichen Phase ein weiterer Angehöriger der Gruppe zu Wort kommt.

\section{Phase VII:}

Die syntaktisch oder semantisch nicht korrekten Übersetzungen werden in Plenum (selbstverständlich mit der Unterstützung und Hilfe des Lehrenden) korrigiert. Die verbesserte Übertragung in die Muttersprache, über die sich die ganze Klasse einig war, wird zum letzten Mal vor der ganzen Klasse vorgetragen.

An dieser Stelle wäre es angebracht, besonders zu unterstreichen, dass sich die Verbesserungen mit Begründung erfolgen. Damit wird gemeint, dass es den Lernenden -auf konkrete Weise- klar gemacht wurde, warum sie bei der Übersetzung manche Fehler begangen hatten. Besteht also der Fehler in der Wortauswahl, in den mangelnden Grammatikkenntnissen? Oder geht es um syntaktische Fehler, da beide Sprachen zu verschiedenen Sprachfamilien gehören?

\section{Phase VIII:}

Nach der Duchführung aller Phasen in den betreffenden Unterrichtseinheiten in Bezug auf die gründliche Behandlug des Textes verlangt man von jedem Studenten, dass er die Nachricht mit eigenen Sätzen mündlich zusammenfasst. Damit die Lerner zur mündlichen Äusserung kommen, ist eine andere Vorgehensweise vorzuschlagen. Dabei werden verschiedene Fragen über den Textinhalt an die ganze Klasse gestellt. Unter Voraussetzung, dass sich jeder auf Deutsch äussert, indem er entweder Fragen stellt oder eine Frage seines Kommilitonen beantwortet, nimmt jeder Lerner aktiv am Unterricht teil.

\section{Phase IV:}

Am Ende der Stunde werden die Gruppen aufgefordert, ähnliche, aktuelle Nachrichten über die Weltereignisse im Internet zu recherchieren und in den Klassenraum mitzubringen.

Jede Nachricht, die zuerst vor der Unterrichtsstunde von der Lehrkraft überprüft und bestätigt wurde, wird in einzelnen Unterrichtsstunden in der Klasse nach den oben genannten Phasen behandelt. Die Lehrkaft begleitet, beobachtet den unterrichtlichen Verlauf und interveniert nur in den nötigen Situationen, falls Wissenslücken entstehen.

Eine andere begleitende Vorgehensweise mit den Nachrichtentexten ist, die zu behandelnde Nachricht durch Bilder, Fotos oder Filme visuell zu unterstützen. Die visuelle Unterstützung spielt auch in der Lektüre einer muttersprachlichen Zeitung eine eminente Rolle, weil sie das Verstehen enorm erleichtert (Hackl - Rössler, 2006, s. 3). So ein Verfahren dient also sowohl zum Vorentlasten des Textes als auch zum Erwecken des Lerninteresses sowie zur Erhöhung der Motivation in den Unterrichtssituationen, wo es sich um die Lektüre einer deutschsprachigen Zeitung oder anderer schriftlichen, fremdsprachlichen Sprachprodukte handelt. Inzwischen wäre es angebracht, nicht ausser Acht zu lassen, dass man eine Fremdsprache dafür zu erlernen versucht, sie in der Zielkultur, also in den realen Sprachsituationen anzuwenden. Wie es auch Çakır $(2017$, s. 86) zum Asdruck gebracht hat, “... könnte man dafür plädieren, authentische Gelegenheiten in den unterrichtlichen Prozess zu integrieren, um fremdsprachenlernende Jugendliche auf die fremdsprachliche Realität vorzubereiten". Auch fremdsprachliche, dem Lernniveau entsprechende Zeitungstexte gehören zu solchen authentischen Texten, die die Lerner auf den Alltag der Zielkultur vorbereiten. 


\section{Schlussbetrachtung}

Es gibt zahlreiche Gründe, die für Vorteile der Verwendung der Meldungen und Nachrichten im Unterrichtsgeschehen sprechen. Diese Vorteile, die zum fremdsprachlichen Lernprozess einen grossen Beitrag leisten, sind wie folgt zu nennen: Die Meldungen und Nachrichten bestehen aus alltäglichen Sätzen, die deshalb als authentisch gelten. Sie bieten eine unendliche Zahl an authentischem Sprachmaterial. Sie sind also Kennzeichen für die Tendenzen der Gegenwartssprache. Es bewirkt selbsverständlich, dass sich die Motivation in der Klasse steigert. Denn der Lerner, der von seinem Alltag aus den muttersprachlichen Zeitungen das behandelnde Thema einigermassen kennt, der also diesbezügliches Vorwissen hat, konzentriert sich nur darauf, wie ein Ereignis fremdsprachlich ausgedrückt wurde, wie es in der Presse der Zielsprache berichtet wurde.

Der Lerner hat ausserdem die Möglichkeit, besonders durch die dasselbe Thema reflektierenden Zeitungstexte die Ausgangskultur mit der Zielkultur zu vergleichen. Es hat zur Folge, dass sich die Kompetenz "Interkulturalität" bei dem Lernenden entwickelt, welche als eines der Hauptziele in der Fremdsprachendidaktik gilt.

Die Behandlung der selben Themen aus verschiedenen Zeitungen hat es zur Folge, dass der Lerner einen noch bereicherten Wortschatz besitzt, weil er mit verschiedenen Wörtern und Wendungen konfrontiert wurde, die eigentlich denselben Inhalt mit verschiedenen Sprachmitteln zur Sprache zu bringen dienen.

Dass der Lerner während des Umgangs mit einer Nachricht auf unbekannte Wörter stösst, bringt auch einen Vorteil mit sich, und zwar einen fremdsprachlich-didaktischen Gewinn. Dass der Gebrauch einsprachiger Wörterbücher (Deutsch-Deutsch) bei dem Entschlüsseln der fremdsprachlichen Texte von der Lehrkraft zugelassen wurde, ermöglicht es gleichzeitig, dass der Lerner auch neue, vorher ihm unbekannte Wörter kennenlernt, während er den Text zu verstehen versucht. Ausserdem hilft die visuelle Unterstützung (Fotos und Bilder, Filmausschnitte usw.) dem Lernenden bei dem Vorentlasten der Nachrichten, die aus komplizierteren Strukturen und Wendungen bestehen. Die eminenten, meist bevorzugten Übungen mit den journalistischen Nachrichten und Meldungen können in Stichpunkten wie folgt aufgezählt werden:

-Schlagzeilen entschlüsseln,

-die muttersprachlichenTexte mit den fremdsprachlichen vergleichen,

-die wichtigsten Fakten aus der Nachricht entnehmen, indem man sich auf die Kernaussagen konzentriert,

-angefangene Nachricht sinngemäss ergänzen,

-mit Hilfe von W- Fragen den Inhalt zusammenfassen (Lothar, 2007, s. 269).

In diesem Zusammenhang erübrigt es sich zu unterstreichen, dass zielsprachige Nachrichten und Meldungen und andere journalistische Texte aus den Zeitungen sowohl zur Förderung des Leseverstehens, als auch zur Entwicklung anderer Grundsprachfertigkeiten beitragen. Inzwischen ist es auch hinzuzufügen, dass das Erreichen kommunikativer Kompetenz in der Fremdsprachendidaktik den Umgang mit authentischen Texten wie jornalistische Sprachmaterialien im fremdsprachlichen Deutschunterricht voraussetzt bzw. zwingt.

\section{QUELLENVERZEICHNIS}

Brinker, K. (2010). Linguistische Textanalyse. Eine Einführung in Grundbegriffe und Methoden.7., durchgesehene Auflage. Berlin: Erich Schmidt. 
Burger, H. (2005). Mediensprache. Eine Einführung in Sprache und Kommunikationsformen der Massenmedien. Mit einem Beitrag von Martin Luginbühl. 3., völlig neu bearbeitete Auflage.Berlin, New York: de Gruyter.

Büyükahıska, D., \& Çebi Kozallık, R. H. (2018).Yabancı Dil Öğrencilerinin Kelime Öğrenme Stratejileri Kullanım S1klıkları. Turkish Studies Educational Sciences Volume 13/19, s. 307-326 DOI Number: http://dx.doi.org/10.7827/TurkishStudies.13939 ISSN: 1308-2140, ANKARATURKEY.

Çakır, G. (2017). Authentizität im Kontext des fremdsprachlichen Lernens, The Journal of Academic Social Science Studies, International Journal of Social Science, Doi number:http://dx.doi.org/10.9761/JASSS7404, Number: 63, s. 83-99.

Gaffarı, R. (2017). Yabancılara Türkçe Öğretiminde Gazete Metinlerinin Kullanımı. Cilt 2, Sayı 1, Haziran 2017. Uluslararası Türkoloji Araştırmaları ve İncelemeleri Dergisi. S. 34-48.

Hackl-Rössler, S. (2006). Textstruktur und Textdesign. Tübingen: Narr.

Lüger, H-H. (1995). Pressesprache. 2., neu bearb. Aufl. Tübingen: Niemeyer.

Jung, L. (2007). 99 Stichwörter zum Unterricht. Deutschland: Max Huber Verlag.

Online I: https://www.welt.de/wissenschaft/umwelt/article154164328/Wassernot-ist-eine-dergroessten-Krisen-unserer-Zeit.html?wtrid=onsite.onsitesearch 